\title{
Physics studies about ECE through an oblique line of sight in ITER and their implications for the detection of neoclassical tearing modes
}

\author{
C. Sozzi" ${ }^{+1}$, L. Figini' , D. Farina' ${ }^{1}$ D. Micheletti', M. A. Henderson ${ }^{2}$ \\ ${ }^{1}$ Istituto di Fisica del Plasma - CNR, Via Roberto Cozzi 53, 20125 Milano, Italy \\ ${ }^{2}$ ITER Organization, 13108 Saint-Paul-lez-Durance, France
}

The alignment of the electron cyclotron waves $(\mathrm{ECW})$ current driven with the radial position of the $\mathrm{O}$ point of magnetic island is one of the key factors in the schemes for Neoclassical Tearing Modes (NTM) control in ITER. Accordingly with the current experience in various tokamaks, the acceptable radial misalignment is about $\Delta \mathrm{R}<1 \mathrm{~cm}$. Such accuracy requires a fine tuned feedback control of the ECW launchers based on a direct measurement of the effectiveness of the injected ECW power. This paper deals with the evaluation of the physics performance of an electron cyclotron emission (ECE) system using the ITER ECH/ECCD Upper Launcher as a receiver line. The concept stems from the so called ECE-in-LINE proposal. This is based on the observation that along a given line of sight, in thermal plasmas, the ECRH power deposition profile is strictly related to the effective ECE profile at the same frequency. The time behavior of the temperature fluctuation associated with the NTMs, observed at ECE frequencies slightly shifted with respect to the injected ECW power measured in a collinear line of sight of ECCD would mark the target volume. A rather simple signal processing would then provide to the control system the information for optimal tracking of the target reducing at a minimum the needed computational steps and consequently the associated uncertainties. While the implementation of a true ECE-in-LINE is technologically challenging and appropriate solutions are still in development, a somewhat rather similar control concept can be warily applied for a quasi-in-line system in which the ECW injection and the ECE reception are toroidally or poloidally shifted. This approach could be implemented in ITER taking advantage of a spare transmission line, provided that the effect of such shift and his dependence on the plasma parameters can be proved weak enough to be neglected or integrated in the control system in all the interesting cases. As a first step of this analysis the spatial resolution for an ITER reference scenario for both 1st harmonics Ordinary Mode and 2nd harmonics Extraordinary Mode emission have been evaluated along the accessible angular range using the SPECE code. This has been translated in quantities useful to define a possible detection and control strategy for the NTMs instabilities. The analysis performed up to now does not show major showstoppers in the evaluation of the feasibility of a quasi-ECE-in-LINE detection system based on the OM1 frequency range. The tangential line of sight with respect to the flux surfaces is affected by limited spatial resolution which can be recovered adopting the strategy of a radial scan which can exploit the intrinsic ECE resolution significantly better.

First EPS Conference on Plasma Diagnostics - $1^{\text {st }}$ ECPD

14-17 April 2015,

Villa Mondragone, Frascati (Rome) Italy

*Speaker. E-mail: sozzi@ifp. cnr.it 


\section{Introduction}

Schemes for NTM suppression using ECCD in feedback control require the integration of several subsystems in a multi-step process aiming to perform the functions that in a coarsegrained description can be listed as: 1) detection of the instability through one or more suitable diagnostics, 2) obtaining its spatial localization, 3) setting the launching coordinates for ECCD launch, and 4) sending the trigger to the gyrotrons controller to switch on/off the EC power. A variety of real time techniques and control strategies are exploited in presently operating devices in order to perform the functions listed above. Generally, step 1 requires the processing of the diagnostics data, steps 2-3 use magnetic equilibrium reconstruction, step 3 requires the computation of the beam refraction, and step 4 may require the computation of phase delay among the instability $\mathrm{O}$ point location and each launcher.
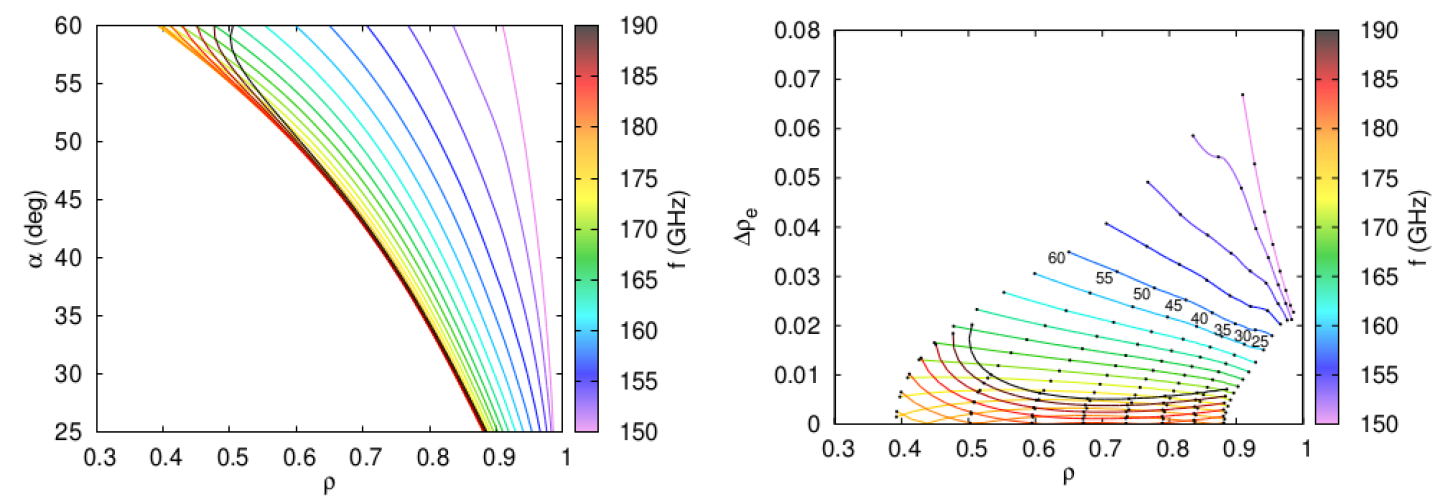

Figure 1. Poloidal steering angle $\alpha$ required to observe a given radial location $\rho$ (left) and variation of the intrinsic radial resolution $\Delta \rho_{\mathrm{e}}$ along the steering (right) at different ECE frequencies. The steering angle $\alpha$ is shown along the curves in the plot at (right) at even steps of $\Delta \alpha=5^{\circ}$.

The alignment of the electron cyclotron waves (ECW) current driven within the $\mathrm{O}$ point of magnetic island is one of the key factors in the schemes for Neoclassical Tearing Modes (NTM) control in ITER. According to the literature, the acceptable radial misalignment is less than 1 $\mathrm{cm}[1,2]$. Such accuracy requires a fine tuned feedback control of the ECW launchers based on a direct measurement of the effectiveness of the injected ECW power. Techniques of alignment based on plasma equilibrium computation only are limited in this respect by the accuracy of the computation itself. The control system needs to optimize both the time and the power needed for the suppression. The required stabilization time includes the time needed for the instability identification (at a size exceeding the minimum detectable size of the diagnostic system), the time for the computation of the launching data, the time for the launching system to realize the requested configuration and the time needed to inject enough energy in the island to suppress or reduce the instability before its evolution towards a locked mode, being the last dependent on the driven current-NTM location alignment. Evaluations for ITER indicate that the time for the mode growth up to locking is about $6 \mathrm{~s}$ and that NTM saturation is possibly never reached [3]. As for the power, the ECH\&CD system will be a multi-tasking actuator in ITER, controlled through a sophisticated system able to manage the different tasks with optimal prioritization and 
time ordering and both the required power as well as the power deployed from other tasks should be considered. For this task, the physics performance of an electron cyclotron emission (ECE) system is investigated using the ITER EC Upper Launcher as a receiver line for the specific purpose of NTM detection and control. The concept stems from the so called ECE-inLINE proposed in [4] and tested experimentally in [5] based on the observation that in thermal plasmas, along a given line of sight, the ECRH power deposition profile is strictly related to the effective ECE profile at the same frequency. A somewhat similar control concept could be applied for a quasi-ECE-in-LINE system in which the ECW injection and the ECE reception are toroidally or poloidally shifted [6]. A similar technique could be implemented in ITER taking advantage of a spare transmission line [7], provided that the effect of such shift and its dependence on plasma parameters can be proved weak enough to be neglected or integrated in the control system in all the interesting cases.
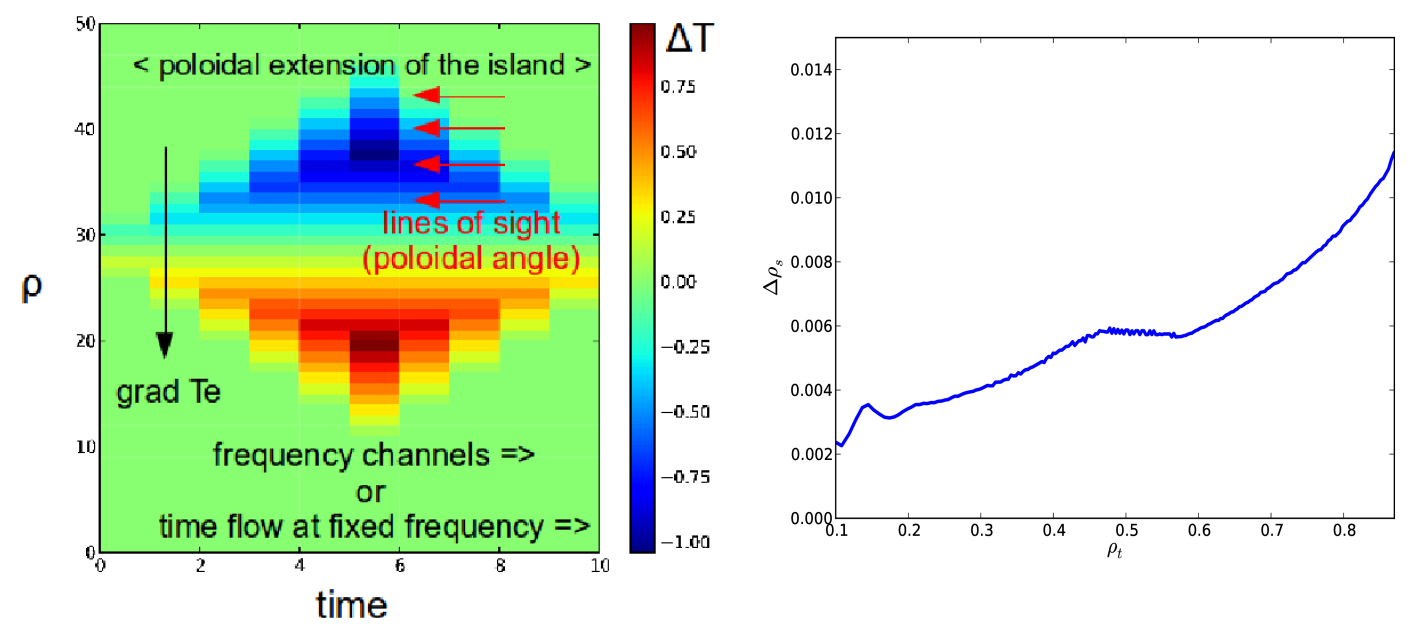

Figure 2. Left: schematic temperature perturbation seen in a tangential line of sight. Right: minimum full-width of a detectable island across its radial location for the assumptions taken about the ECE sensitivity $(100 \mathrm{eV})$.

\section{ECE sensitivity and implications for the NTM control in ITER}

The spatial resolution for a ITER reference scenario of both 1 st harmonics ordinary mode (OM1) and 2nd harmonics extraordinary mode (XM2) emission has been evaluated along the accessible angular range using the SPECE code [8] to assess a possible detection strategy to be used for NTM control purposes. Figure 1 (left) shows the radial range that can be achieved by varying the poloidal steering angle $\alpha$ when observing OM1 emission at different frequencies, and (right) the radial extent $\Delta \rho_{\mathrm{e}}$ of the emission if the transverse beam size is neglected. The poloidal and toroidal receiving (or launching) angles $\alpha, \beta$ are defined in terms of the cylindrical components of the wavevector $\mathrm{N}_{\mathrm{R} 0}=-\cos \beta \cos \alpha, \mathrm{N}_{\varphi 0}=\sin \beta, \mathrm{N}_{\mathrm{Z} 0}=-\cos \beta \sin \alpha$, with $-180^{\circ}$ $\leq \alpha \leq 180^{\circ}$, and $-90^{\circ} \leq \beta \leq 90^{\circ}$. In the frequency range $170 \mathrm{GHz} \leq \mathrm{f} \leq 190 \mathrm{GHz}$ the radial range reaches its maximum extension towards the plasma center and the intrinsic radial resolution is minimized. The UL geometry is designed so that the EC beam is close to tangency to the flux surfaces for ECCD at $170 \mathrm{GHz}$. As a consequence the line of sight of an ECE system viewing through a spare line of the UL would cross the island nearly perpendicularly with respect to the 
temperature gradient (figure 2, left), and with a non negligible toroidal component being $\beta=20^{\circ}$. This layout impacts on the waveform of the measurable signals (and on the detection algorithm) and on the spatial resolution. Each signal shows a "unipolar" variation so that the island detection requires the comparison of a set of $2 n+1(n=2,3)$ signals centered on the O-point and spatially or frequency shifted. Nearly equatorial line of sight systems require a single line of sight and GHz-sized spacing of the frequency channels. A typical sensitivity $\Delta \mathrm{T}_{\mathrm{s}}=100 \mathrm{eV}$ has been used as a reference value for the ECE detection system, based on the conservative assumption of 5-10 eV detector sensitivity, limited by its noise temperature, and of $\sim 30 \mathrm{eV}$ plasma thermal noise at $\rho \sim 0.8,2 \mathrm{GHz}$ bw, $10 \mathrm{kHz}$ low pass filter [9,10]. NTM instabilities can thus be detected with ECE if the associated magnetic islands cause a fluctuation in the ECE signal $\Delta \mathrm{T}>\Delta \mathrm{T}_{\mathrm{s}}$ when they cross the ECE line of sight (figure 2, right). In a simplified approach, assuming a uniform temperature profile inside the island and a temperature profile across the island $\mathrm{X}$-point equal to the unperturbed profile, one can estimate the minimum island half-width $\Delta \rho_{\mathrm{s}}$ that an island at radial location $\rho$ must have to be detected, as $\Delta \rho_{\mathrm{s}}=\Delta \mathrm{Ts} /$ $(\mathrm{dT} / \mathrm{d} \rho)$. The temperature gradient in this plasma scenario is of the order of $\mathrm{dT} / \mathrm{d} \rho \sim 10-20 \mathrm{keV}$, so that the threshold for the island width is about $\Delta \rho_{\mathrm{s}} \sim 0.005-0.01$ over most of the plasma radial extent, corresponding to a full width $\mathrm{w}=2 \mathrm{a} \Delta \rho_{\mathrm{s}} \sim 2-4 \mathrm{~cm}$, being a $=2 \mathrm{~m}$ the plasma minor radius.
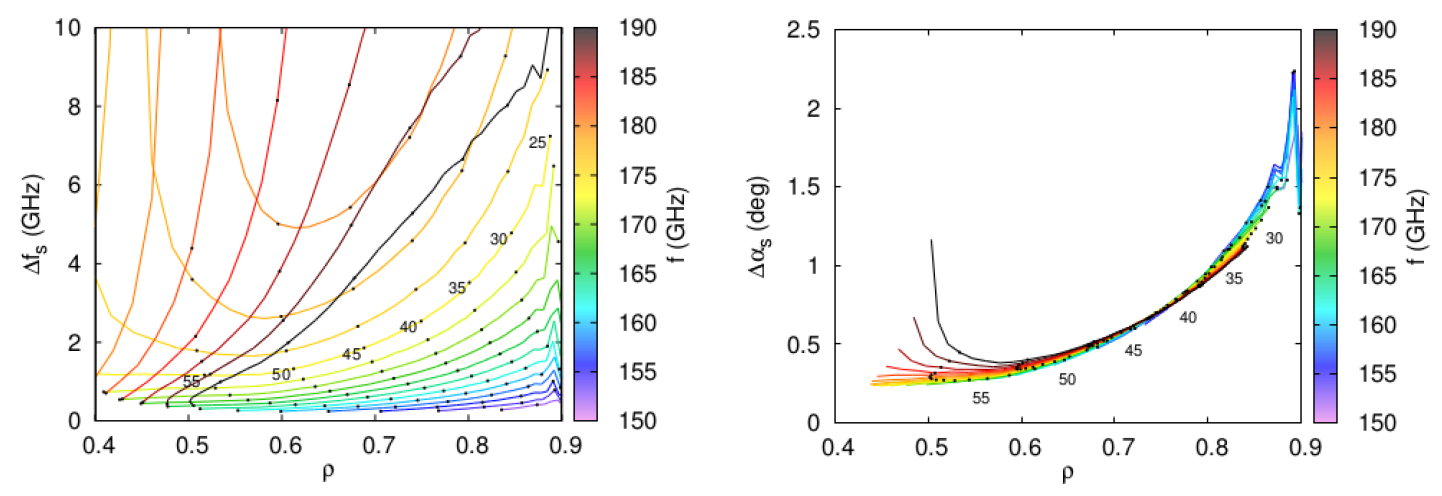

Figure 3. OM1 sensitivity to changes in frequency along the line of sight (left), and in observation angle (right). The frequency is represented through the color scale and the black dots mark the contours of constant angle of observation $\alpha$.

\section{ECE modeling}

Two quantities can be introduced to express the sensitivity to the frequency and to the observation angle, which may help to design the control system. In order to shift the median emission radius $\Delta \rho_{50}$ by an extent $\Delta \rho_{\mathrm{s}}$, a change in frequency $\Delta \mathrm{f}_{\mathrm{s}}=\Delta \rho_{\mathrm{s}}\left(\mathrm{d} \rho_{50} / \mathrm{df}\right)^{-1}$ or a change in poloidal angle $\Delta \alpha_{\mathrm{s}}=\Delta \rho_{\mathrm{s}}\left(\mathrm{d} \rho_{50} / \mathrm{d} \alpha\right)^{-1}$ are necessary. These quantities allow configuring the ECE diagnostics (frequency spacing, number of frequency channels) and the optimal detection strategy (number of lines of sight and scanning range) according to the receiver sensitivity (which determines $\Delta \rho_{s}$ ), and keeping into account the expected latency in the measurement, not discussed in this paper [11]. Figure 3 shows the OM1 sensitivity to changes in frequency along the line of sight (left), and in observation angle (right). The frequency is represented through the 
color scale and the black dots mark the contours of constant angle of observation $\alpha$. It is shown that there is a loss of sensitivity (in frequency) towards the frequency value for which tangency to the flux surface at the resonance is achieved: at a given radial location a smaller $\Delta \mathrm{f}_{\mathrm{s}}$ is obtained by choosing an observation angle (contours marked by black dots) - frequency (color coding) combination far from tangency, i.e. with extreme frequency values, either very low or very high. The practical implication of this on the problem of the NTM location detection by comparing the ECE emission at various frequencies is that such process is strongly dependent from the observation angle $\alpha$, and then the optimal spacing among the detection channels could hardly be selected. Conversely, the angular resolution $\Delta \alpha_{\mathrm{s}}$ is quite independent from the anglefrequency combination chosen to reach a particular radial location: its value is mainly determined by geometrical considerations, i.e. by the distance of the emission volume from the steering mirror. At large radii $\rho$ the emission occurs closer to the mirror and a larger steering is required to span a given spatial extent in the plasma. In particular, all the radial locations with $\rho>0.55$ can be observed at any frequency in the range $165-185 \mathrm{GHz}$ only controlling the observation angle.

The ITER ECH/ECCD Upper Launcher steering mirror is able to scan the launched beam at an angular speed of $6 \% \mathrm{sec}=|\delta \mathrm{R} / \delta \mathrm{t}|=1.7 \mathrm{~cm} / 0.1 \mathrm{~s}$ at the $\mathrm{q}=2$ radius, i.e. $\rho \sim 0.8$, with a minimal step size of $0.07 \mathrm{~cm} \mathrm{[12].} \mathrm{The} \mathrm{maximum} \mathrm{acceptable} \mathrm{size} \mathrm{for} \mathrm{a} \mathrm{NTM} \mathrm{island} \mathrm{in} \mathrm{ITER} \mathrm{is} \mathrm{w}=5 \mathrm{~cm}$ [10]. Assuming to use one of the ITER ECH/ECCD Upper Launcher steering mirrors to scan the radial length corresponding to such island size, the required scanning time would be $\sim 0.6 \mathrm{sec}$. This time should be compared with the expected time for a uncontrolled island to grow ( $\sim 6 \mathrm{~s})$. A rather better performance with the reduction of the scanning time could be obtained using two slightly different lines of sight separated by $\Delta \alpha=\mathrm{k} \quad \Delta \alpha_{\mathrm{s}}$ where $0.5 \leq \mathrm{k} \leq 1$ for the simultaneous detection along two angles.

\section{Discussion, conclusions and future work.}

The analysis performed up to now does not show major showstoppers in the evaluation of the feasibility of a quasi-ECE-in-LINE detection system based on the OM1 frequency range. The problem of the reduced spatial resolution along the line of sight due to tangential view with respect to the flux surfaces (which is intrinsicaly related with the oblique line of sight) can be overcome adopting the strategy of a radial scan obtained with the poloidal steering which can exploit the intrinsic ECE resolution significantly better than the frequency-related detection which is strongly dependent from the observation angle. The radial scan can be performed with "oscillations" of the detecting mirror around the expected radial location of the island O-point and/or using more than one line of sight at slightly different poloidal angles.

The quasi-ECE-in-LINE scheme is based on strict relation between the ECRH/ECCD power deposition profile and the ECE profile at the same frequency. For the ITER UL case if the two lines of sight belong to the same launcher and to different transmission lines rows the poloidal offset between injection/observation angle is $\sim 7^{\circ}$ (USM/LSM separation $\sim 25 \mathrm{~cm}$ for a distance between the last mirror and the EC resonance of $\sim 2 \mathrm{~m}$ ). The question arises if the offset due from the poloidal shift can be considered as a constant within the resolution limits, or if and how much its variation can be described by a simple function of $(\alpha, f)$. This will be 
subject of a further investigation. Moreover the dependence on the plasma shape (different scenarios) should be assessed to decide if a single "fitting" function would be sufficient to describe such offset.

The extension of the concept to the case in which the receiver is placed in another toroidal sector with respect to the active ECH/ECCD launcher should also be considered.

Finally, the impact of the finite size of the beam, resulting in the poloidal broadening of the receiver beam on the spatial resolution needs a further assessment.

No advantages and some additional limitations appear from the choice of the XM2 , mainly due to the parasitic third harmonics emission along the ECE path towards the antenna.

\section{References}

[1] S. Cirant et al, Mode Coupling Trigger of Tearing Modes in ECW Heated Discharges in FTU Tokamak, $18^{\text {th }}$ International Atomic Energy Agency Fusion Energy Conference, Sorrento, Italy, 4-10 October 2000 IAEA-FEC, Paper IAEA-CN77/EX3/3 (2001)

[2] E.Kolemen et. al., State-of-the-art neoclassical tearing mode control in DIII-D using real-time steerable electron cyclotron current drive launchers, Nucl. Fusion 54073020 (2014)

[3] R.J. La Haye et al., Prospects for stabilization of neoclassical tearing modes by electron cyclotron current drive in ITER, Nucl. Fusion 49 (2009) 045005 (8pp)

[4] E. Westerhof et al, A generic method for controlled ECRH/ECCD localization $13^{\text {th }}$ Joint Workshop on ECE \& ECRH (17-20 May 2004), (2004) p. 357

[5] B.A. Hennen et al., Real-time control of tearing modes using a line-of-sight electron cyclotron emission diagnostic, Plasma Phys. Control. Fusion 52 (2010), 104006 (20pp)

[6] F.A.G. Volpe et al., Advanced techniques for neoclassical tearing mode control in DIII-D, Phys Plasmas 16, 102502 (2009)

[7] M.A. Henderson et al, An overview of the ITER ECH\&CD system and functional capabilities, $34^{\text {th }}$ Int. Conf. On Infrared, Millimeter and Terahertz waves, Busan South- Korea, 898-901, IEEE 10.1109/ICIMW.2009.5325524 (2009)

[8] D. Farina et al, SPECE: a code for Electron Cyclotron Emission in tokamaks, Int. Conf. Burning Plasma Diagnostics, 24-28 September 2007, Varenna (Italy) AIP Conf. Proc., vol. 988, p. 128 (2008)

[9] Austin, M.E., Resolution of Electron Cyclotron Emission measurements of magnetohydrodynamic modes in ITER, Fusion Science and Technology Vol. 59 (2011), Issue 4, 647-650

[10] V.S. Udintsev, Exploring measurement capabilities of ITER ECE system, IAEA-Fusion Energy Conference 2012, Proc. FEC (San Diego) IAEA-CN-197/ITR/P5-41 (2012)

[11] H. van der Brand et al, Evaluating neoclassical tearing mode detection with ECE for control on ITER, Nucl. Fusion 53 (2013) 013005 (13pp)

[12] A. Collazos, et al., Progress on the ITER ECRH upper launcher steering mirror identification and control, Fusion Engineering and Design 84 (2009), 618-622 